\title{
HIV-positive Raucher sterben heute eher an Lungenkrebs als an AIDS
}

\begin{abstract}
Antiretroviral behandelte HIV-Patienten, die das Rauchen nicht lassen können, versterben bis zu 13-mal häufiger an Lungenkrebs als an einem AIDSbedingten Leiden. Deshalb sollte die Raucherentwöhnung ein wesentlicher Bestandteil der Therapie sein.
\end{abstract}

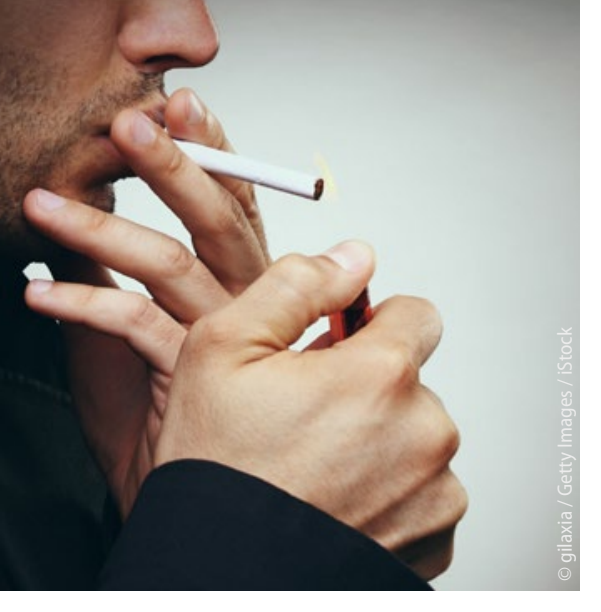

Vor Einführung der antiretroviralen Therapie (ART) lebten HIV-Infizierte nicht lange genug, um an Lungenkrebs zu sterben. Das hat sich geändert, seit das HI-Virus medikamentös erfolgreich in Schach gehalten werden kann.

Krishna Reddy und Kollegen vom Massachusetts General Hospital in Boston haben mithilfe eines Mikrosimulationsmodells den Einfluss des Tabakkonsums auf die Lungenkrebssterblichkeit von HIV-Infizierten untersucht.

Die kumulative Lungenkrebsmortalität 40-jähriger Männer unter ART, die weiterhin rauchen, erreicht danach bis zum 80. Lebensjahr für starke Raucher (35 Zigaretten/Tag) 28,9\%, für moderate (18/Tag) $23,0 \%$ und für Wenigraucher (2/Tag) 18,8\%. Bei HIV-positiven Männern, die nie geraucht haben, liegt die Quote bei 1,6\%. Ein Rauchstopp im Alter von 40 Jahren würde die Mortalitätsraten auf $7,9 \%$ bzw. $6,1 \%$ bzw. $4,3 \%$ sen- ken. Für HIV-positive Frauen errechneten sich Quoten von 27,8\% bzw. 20,9\% bzw. 16,6\% bzw. 1,2\% und für Patientinnen, die das Rauchen mit 40 aufgegeben haben, von 7,5\% bzw. 5,2\% bzw. 3,7\%.

Bei Männern, die unter der ART moderat weiterrauchen, ist die kumulative Lungenkrebsmortalität zehnmal so hoch wie die Sterblichkeit infolge AIDSspezifischer Ursachen, bei Frauen erreicht sie das Achtfache.

Basierend auf diesen Hochrechnungen, so Reddy und Kollegen, sei davon auszugehen, dass 9,3\% der etwa 644.200 HIV-infizierten, therapierten US-Amerikaner zwischen 20 und 64 Jahren an Lungenkrebs sterben werden - es sei denn, sie hören auf zu rauchen.

Dr. Christine Starostzik

- Reddy KP et al. AMA Internal Medicine 2017; online 18. Sept. 2017

https://jamanetwork.com/journals/jamainternalmedicine/ article-abstract/2653450

\section{Regelmäßige Kontrollen nötig}

\section{Cave: HIV-assoziierte kognitive Störungen}

\section{Damit neurokognitive Störungen bei HIV-Patienten früh erkannt werden, sind wiederholte Überprüfungen der entsprechenden Funktionen nötig.}

_ In einer prospektiven Studie haben Ei Kinai vom AIDS Clinical Center in Tokio und Kollegen 728 HIV-infizierte Patienten im Durchschnittsalter von 45 Jahren untersucht. Gemäß den „Frascati“-Kriterien wurden HIV-assoziierte neurokognitive Störungen (HAND) der Probanden erfasst. Neuropsychologische Funktionstests zur Überprüfung exekutiver und visuell- räumlicher Aufgaben bewährten sich dabei zur Diagnose einer HAND am besten.

Bei 25,3\% der Patienten diagnostizierten Kinai und Kollegen eine HAND (13,5\% asymptomatische neurokognitive Beeinträchtigungen; 10,6\% leichte neurokognitive Störungen, MND; $1,2 \%$ HIV-assoziierte Demenz, HAD). In der multivariaten Analyse wurden ein Alter ab 50 Jahren (OR 2,2) sowie eine unvollständige Virussuppression (OR 1,96) als Risikofaktoren symptomatischer kognitiver Beeinträchtigungen identifiziert. Eine aktuelle antiretrovirale Therapie dagegen erwies sich als protektiver Faktor $(\mathrm{OR} 0,57)$. MND und HAD traten in den ersten zwei bis sechs Jahren der Infektion signifikant seltener auf als in einem späteren Stadium (6,3\% vs. $17,3 \%$ ab dem elften Jahr). Außerdem zeigten sich neurokognitive Beeinträchtigungen bei Patienten ab 50 häufiger als bei Jüngeren, selbst in frühen Infektionsstadien. Kinai und Kollegen werten diese Befunde als weiteres Argument für einen frühen Therapiebeginn.

- Kinai E. et al. J. Neurovirol. 2017; online 2. Okt. 2017 https://link.springer.com/article/10.1007/s13365-017-0580-6 